\title{
A NOTE ON STRONG SOLUTIONS OF STOCHASTIC DIFFERENTIAL EQUATIONS WITH A DISCONTINUOUS DRIFT COEFFICIENT
}

\author{
NIKOLAOS HALIDIAS AND P. E. KLOEDEN
}

Received 11 November 2004; Revised 20 September 2005; Accepted 21 September 2005

The existence of a mean-square continuous strong solution is established for vectorvalued Itô stochastic differential equations with a discontinuous drift coefficient, which is an increasing function, and with a Lipschitz continuous diffusion coefficient. A scalar stochastic differential equation with the Heaviside function as its drift coefficient is considered as an example. Upper and lower solutions are used in the proof.

Copyright (C) 2006 N. Halidias and P. E. Kloeden. This is an open access article distributed under the Creative Commons Attribution License, which permits unrestricted use, distribution, and reproduction in any medium, provided the original work is properly cited.

\section{Introduction}

Existence theorems [9-12] for Itô stochastic differential equations

$$
d X_{t}=f\left(t, X_{t}\right) d t+g\left(t, X_{t}\right) d W_{t}, \quad t \in[0, T]
$$

usually require that the drift and diffusion coefficients, $f$ and $g$, be at least continuous (in $x$ ) as well as satisfying a growth condition to prevent explosions. An example of Tanaka (e.g., [12, page 71]) with zero drift and a discontinuous diffusion coefficient is known to have no strong solution with zero initial value that is a solution corresponding to a specified Wiener process in contrast to a weak solution where some other Wiener process could be used. Moreover, Barlow [2] shows that a strong solution need not exist when the diffusion process is only continuous. Krylov [7] and Krylov and Liptser [8] (see also the references cited therein) have investigated existence issues for SDE with discontinuous coefficients.

In contrast, here we consider the existence of mean-square continuous strong solutions with a Lipschitz continuous diffusion coefficient but a discontinuous drift coefficient, such as in the scalar SDE

$$
d X_{t}=H\left(X_{t}\right) d t+d W_{t}
$$

Hindawi Publishing Corporation Journal of Applied Mathematics and Stochastic Analysis Volume 2006, Article ID 73257, Pages 1-6 DOI 10.1155/JAMSA/2006/73257 
where $H: \mathbb{R} \rightarrow \mathbb{R}$ is the Heaviside function, which is defined by

$$
H(x):= \begin{cases}0, & x<0 \\ 1, & x \geq 0\end{cases}
$$

Such equations arise, for example, when one considers the effects of background noise on switching systems or other discontinuous ordinary differential equations [5].

Several possible methods could be used here. Following Krylov, one could approximate the drift coefficient by a sequence of smooth functions (e.g., sigmoidal-shaped functions in the case of the Heaviside function). Alternatively, one could reformulate the equation as a stochastic differential inclusion. However, here we will use a method based on upper and lower solutions of stochastic differential equations.

\section{Upper and lower solutions}

We consider an Itô stochastic differential equation

$$
d X_{t}=f\left(t, X_{t}\right) d t+g\left(t, X_{t}\right) d W_{t}, \quad t \in[0, T]
$$

with coefficients $f:[0, T] \times \mathbb{R}^{d} \rightarrow \mathbb{R}^{d}$ and $g:[0, T] \times \mathbb{R}^{d} \rightarrow \mathbb{R}^{d \times k}$, where $W_{t}$ is a given $k$-dimensional Wiener process.

Let $\left(\Omega, \mathscr{F}_{F}, P\right)$ be a complete probability space and let $\left\{\mathscr{F}_{t}\right\}_{t \geq 0}$ be the smallest filtration generated by the Wiener process $W_{t}$.

By a strong solution of the $\operatorname{SDE}(2.1)$ on an interval $[0, T]$ we mean a stochastic process $X_{t}$ which is $\mathscr{F}_{t}$-measurable for each $t \in[0, T]$ with $\mathbb{E}\left\|X_{t}\right\|^{2}<\infty$ for all $t \in[0, T]$ such that

$$
X_{t}=X_{0}+\int_{0}^{t} f\left(s, X_{s}\right) d s+\int_{0}^{t} g\left(s, X_{s}\right) d W_{s}, \quad t \in[0, T], \text { w.p.1. }
$$

(It is assumed implicitly that the integrals on the right-hand side exist w.p.1.) Such a strong solution is sample-path continuous when the coefficients $f$ and $g$ are sufficiently regular, for example, satisfy a global Lipschitz condition.

Upper and lower solutions of an SDE (2.1) have been considered previously under other names in $[1,9]$. Conditions ensuring their existence were given in [1] and they were used in the context of comparison theorems in [9] (see also [3]).

Definition 2.1. A $\mathscr{F}_{t}$-measurable stochastic process $Z_{t}$ is an upper solution of the SDE (2.1) on the interval $[0, T]$ if the inequality (interpreted component wise)

$$
Z_{t} \geq Z_{0}+\int_{0}^{t} f\left(s, Z_{s}\right) d s+\int_{0}^{t} g\left(s, Z_{s}\right) d W_{s}, \quad t \in[0, T]
$$

holds with probability 1 . If $Z_{t}$ satisfies the reversed inequality (i.e., with $\leq$ ), then $Z_{t}$ is a lower solution.

Upper and lower solutions provide useful bounds on strong solutions of an initial value problem for an SDE (2.1) and are often easier to determine explicitly. In the following theorem we show that a strong solution lying between lower and upper solutions 
exists in a special case which suffices later to prove the existence of a strong solution of an SDE with a discontinuous drift coefficient. (Analogous definitions of upper, lower, and strong solutions hold if the drift or diffusion coefficient is nonanticipatively random.)

Our first theorem can be considered as a comparison result that we will need in our main result, that is, Theorem 3.1.

Theorem 2.2. Suppose that the mapping $f:[0, T] \times \Omega \rightarrow \mathbb{R}^{d}$ is measurable and nonanticipative with $\int_{0}^{T} \mathbb{E}\|f(t, \cdot)\|^{2} d t<\infty$, that $g:[0, T] \times \mathbb{R}^{d} \rightarrow \mathbb{R}^{d \times k}$ is Lipschitz continuous and satisfies the linear growth bound

$$
\|g(t, x)\| \leq K+L\|x\|, \quad t \in[0, T], x \in \mathbb{R}^{d}
$$

and that $Z_{t}$ and $Y_{t}$ are upper and lower solutions of the SDE

$$
d X_{t}=f(t, \omega) d t+g\left(t, X_{t}\right) d W_{t}, \quad t \in[0, T]
$$

on $[0, T]$ with $\mathbb{E}\left\|Y_{t}\right\|^{2}<\infty, \mathbb{E}\left\|Z_{t}\right\|^{2}<\infty$, and $Y_{t} \leq Z_{t}$ for $t \in[0, T]$, w.p.1. In addition, suppose that $X_{0}$ is $\mathscr{F}_{0}$-measurable with $\mathbb{E}\left\|X_{0}\right\|^{2}<\infty$ and $Y_{0} \leq X_{0} \leq Z_{0}$.

Then there exists a unique pathwise continuous strong solution $X_{t}$ which satisfies $Y_{t} \leq$ $X_{t} \leq Z_{t}$ for $t \in[0, T]$, w.p. 1 .

Proof. We define the functions $p, r:[0, T] \times \mathbb{R}^{d} \times \Omega \rightarrow \mathbb{R}^{d}$ by

$$
\begin{gathered}
p(t, x, \omega):=\max \left\{Y_{t}(\omega), \min \left\{Z_{t}(\omega), x\right\}\right\}, \\
r(t, x, \omega):=\frac{p(t, x, \omega)-x}{1+\|x\|^{2}},
\end{gathered}
$$

and consider the stochastic differential equation

$$
d X_{t}=\left(f(t, \omega)+r\left(t, X_{t}, \omega\right)\right) d t+g\left(p\left(t, X_{t}, \omega\right)\right) d W_{t}, \quad t \in[0, T]
$$

for the given initial condition $X_{0}$. This SDE has nonanticipative random coefficients

$$
\begin{gathered}
\tilde{f}(t, x, \omega):=f(t, \omega)+r(t, x, \omega), \\
\tilde{g}(t, x, \omega):=g(p(t, x, \omega)),
\end{gathered}
$$

which are Lipschitz continuous in $x$ and satisfy a growth bound of the form

$$
\|\tilde{f}(t, \omega)\|+\|\tilde{g}(t, x, \omega)\| \leq K\left(1+\max \left\{\|f(t, \omega)\|,\left\|Y_{t}(\omega)\right\|,\left\|Z_{t}(\omega)\right\|\right\}\right)=: \tilde{K}_{t}(\omega),
$$

where the $\tilde{K}_{t}$ is nonanticipative with $\int_{0}^{T} \mathbb{E} \widetilde{K}_{s}^{2} d s<\infty$. Thus, from [6, Chapter 5, Theorem 2.9] the SDE (2.5) has a unique strong solution $X_{t}$, which is pathwise continuous. (The solution is also mean-square continuous, which is shown within the proof and is what we need in the sequel.) 
We will now show that $Y_{t} \leq X_{t} \leq Z_{t}$ for $t \in[0, T]$, w.p.1. Suppose that there exists an interval $\left(t_{1}, t_{2}\right) \subset[0, T]$ such that $X_{t_{1}}=Y_{t_{1}}$ and $X_{t} \leq Y_{t}$ for $t \in\left(t_{1}, t_{2}\right)$. Then

$$
X_{t}-Y_{t} \geq \int_{t_{1}}^{t} r\left(s, X_{s}, \omega\right) d s+\int_{t_{1}}^{t}\left(g\left(p\left(s, X_{s}, \omega\right)\right)-g\left(Y_{s}\right)\right) d W_{s}=\int_{t_{1}}^{t} r\left(s, X_{s}, \omega\right) d s \geq 0
$$

for all $t \in\left(t_{1}, t_{2}\right)$, since $p\left(t, X_{t}, \omega\right) \equiv Y_{t}$ in $\left(t_{1}, t_{2}\right)$, so $r\left(t, X_{t}, \omega\right)=\left(Y_{t}-X_{t}\right) /\left(1+\left\|X_{t}\right\|^{2}\right) \geq 0$, which gives a contradiction. Thus, $X_{t} \geq Y_{t}$ for all $t \in[0, T]$. Using the same argument we can also prove that $X_{t} \leq Z_{t}$. That is, $X_{t}$ is in fact a strong solution of the original SDE (2.5).

\section{SDEs with discontinuous drift coefficient}

We now restrict attention to the autonomous SDE

$$
d X_{t}=f\left(X_{t}\right) d t+g\left(X_{t}\right) d W_{t}, \quad t \in[0, T]
$$

and assume that the drift coefficient is increasing, that is, $f(x) \leq f(y)$ whenever $x \leq y$ (where the inequalities are interpreted componentwise), but need not be continuous. In addition, we assume that the diffusion coefficient is Lipschitz continuous. This applies in particular to the scalar SDE (1.2) with the Heaviside drift coefficient. We show that the SDE (3.1) has a strong solution whenever it has an upper and a lower solution.

Theorem 3.1. Suppose that $f, g: \mathbb{R}^{d} \rightarrow \mathbb{R}^{d}$ and $g: \mathbb{R}^{d} \rightarrow \mathbb{R}^{d \times k}$ both satisfy the linear growth bound (2.4) and, in addition, that $f$ is increasing and $g$ is Lipschitz continuous. Moreover, suppose that the SDE (3.1) has mean-square continuous upper and lower solutions $Z_{t}$ and $Y_{t}$ on $[0, T]$ with $\int_{0}^{T} \mathbb{E}\left\|f\left(Y_{t}\right)\right\|^{2} d t<\infty, \int_{0}^{T} \mathbb{E}\left\|f\left(Z_{t}\right)\right\|^{2} d t<\infty$, and $Y_{t} \leq Z_{t}$ for $t \in[0, T]$, w.p. 1 .

Then the SDE (3.1) has at least one mean-square continuous strong solution $X_{t}$. Moreover, $Y_{t} \leq X_{t} \leq Z_{t}$ for $t \in[0, T]$,w.p.1.

Proof. We define by $\mathscr{C}$ the space of all $d$-dimensional nonanticipative mean-square continuous stochastic process $X=\left\{X_{t}, t \in[0, T]\right\}$ satisfying $\sup _{0 \leq s \leq T} \mathbb{E}\left\|X_{s}\right\|^{2}<\infty$ with the norm $\|X\|:=\left(\sup _{0 \leq s \leq T} \mathbb{E}\left\|X_{s}\right\|^{2}\right)^{1 / 2}$, which is a Banach space.

We denote by $\mathscr{K}$ the order interval $[Y, Z]$ in $\mathscr{X}$, that is, consisting of all $X$ in $\mathscr{L}$ with $Y_{t} \leq X_{t} \leq Z_{t}$ for $t \in[0, T]$, w.p.1, which is closed and bounded in the above norm. Using the Lebesgue dominated convergence theorem, one can prove that a monotone sequence that belongs to $\mathscr{K}$ converges in $\mathscr{X}$. Thus, $\mathscr{K}$, with the above norm, is a regularly ordered metric space (for the definition, see [4, page 117]).

For any process $U \in \mathscr{K}$, it is clear that $Y$ and $Z$ are also mean-square continuous lower and upper solutions for the SDE

$$
d X_{t}=f\left(U_{t}(\omega)\right) d t+g\left(X_{t}\right) d W_{t}, \quad t \in[0, T]
$$

Thus, by Theorem 2.2, for any $\mathscr{F}_{0}$-measurable $X_{0}$ with $\mathbb{E}\left\|X_{0}\right\|^{2}<\infty$ and $Y_{0} \leq X_{0} \leq Z_{0}$, the $\operatorname{SDE}$ (3.2) has a mean-square continuous (in fact, pathwise continuous) unique strong solution $X_{t}$, which satisfies $Y_{t} \leq X_{t} \leq Z_{t}$ for all $t \in[0, T]$, w.p.1. 
We define an operator $S: \mathscr{K} \rightarrow \mathscr{K}$ where $X=S(U)$ is the unique mean-square continuous strong solution of the $\operatorname{SDE}(3.2)$ corresponding to the stochastic process $U \in \mathcal{Y}$. We will apply [4, Corollary 3.2] to show that $S$ has a fixed point, which is then the desired solution. For this we only have to prove that $S$ is an increasing map.

We will prove that if $U^{(1)}$ and $U^{(2)}$ are stochastic processes in $\mathscr{K}$ with $U_{t}^{(1)} \leq U_{t}^{(2)}$ for all $t \in[0, T]$ and if $X^{(1)}=S\left(U^{(1)}\right), X^{(2)}=S\left(U^{(2)}\right)$, then $X_{t}^{(1)} \leq X_{t}^{(2)}$ for all $t \in[0, T]$.

Let us choose stochastic processes $U^{(1)}, U^{(2)}$ in $\mathscr{K}$ with $U_{t}^{(1)} \leq U_{t}^{(2)}$ for all $t \in[0, T]$ and define $X^{(1)}=S\left(U^{(1)}\right)$. Since the drift coefficient $f$ is an increasing function, $X_{t}^{(1)}$ is a lower solution of the SDE

$$
X_{t}=X_{0}+\int_{0}^{t} f\left(U_{s}^{(2)}\right) d s+\int_{0}^{t} g\left(X_{s}\right) d W_{s}
$$

But this problem has an upper solution, namely, the stochastic process $Z_{t}$. Thus, by Theorem 2.2, the SDE (3.3) has a mean-square continuous strong solution $X_{t}^{(2)}$, which satisfies $X_{t}^{(1)} \leq X_{t}^{(2)} \leq Z_{t}$. Now $X^{(2)}=S\left(U^{(2)}\right)$, so $S$ is an increasing map as required and thus, by $\left[4\right.$, Corollary 3.2], has a fixed point $X^{*}=S\left(X^{*}\right) \in \mathscr{K}$, that is,

$$
X_{t}^{*}=X_{0}+\int_{0}^{t} f\left(X_{s}^{*}\right) d s+\int_{0}^{t} g\left(X_{s}^{*}\right) d W_{s}
$$

with $Y_{t} \leq X_{t}^{*} \leq Z_{t}$ for all $t \in[0, T]$, w.p.1. In particular, $X_{t}^{*}$ is nonanticipative and meansquare continuous.

We can apply Theorem 3.1 to the scalar SDE (1.2) with the Heaviside drift coefficient $f(x)=H(x)$ and diffusion coefficient $g(x) \equiv 1$. First we note that $H(x)$ is an increasing function and then that

$$
X_{0}+\int_{0}^{t} d W_{s} \leq X_{0}+\int_{0}^{t} H\left(X_{s}\right) d s+\int_{0}^{t} d W_{s} \leq X_{0}+\int_{0}^{t} 1 d s+\int_{0}^{t} d W_{s}
$$

for any sample path continuous, nonanticipative stochastic process $X_{t}$. Hence $Y_{t}:=X_{0}+$ $W_{t}$ and $Z_{t}:=X_{0}+W_{t}+t$ are lower and upper solutions for the Heaviside SDE (1.2). Thus the Heaviside SDE (1.2) has at least one mean-square continuous strong solution $X_{t}^{*}$ taking values between those of these lower and upper solutions, specifically with

$$
X_{0}+W_{t} \leq X_{t}^{*} \leq X_{0}+1+W_{t}, \quad t \in[0, T], \text { w.p.1. }
$$

\section{Acknowledgment}

We would like to thank Professor Y. Ouknine for his helpful criticism of an earlier version of this paper.

\section{References}

[1] S. Assing and R. Manthey, The behavior of solutions of stochastic differential inequalities, Probability Theory and Related Fields 103 (1995), no. 4, 493-514.

[2] M. T. Barlow, One-dimensional stochastic differential equations with no strong solution, The Journal of the London Mathematical Society. Second Series 26 (1982), no. 2, 335-347. 


\section{SDEs with discontinuous drift}

[3] I. Chueshov, Monotone Random Systems - Theory and Applications, Lecture Notes in Mathematics, vol. 1779, Springer, Berlin, 2002.

[4] S. Heikkilä and S. Hu, On fixed points of multifunctions in ordered spaces, Applicable Analysis $\mathbf{5 1}$ (1993), no. 1-4, 115-127.

[5] S. Heikkilä and V. Lakshmikantham, Monotone Iterative Techniques for Discontinuous Nonlinear Differential Equations, Monographs and Textbooks in Pure and Applied Mathematics, vol. 181, Marcel Dekker, New York, 1994.

[6] I. Karatzas and S. Shreve, Brownian Motion and Stochastic Calculus, Springer, New York, 1991.

[7] N. V. Krylov, On weak uniqueness for some diffusions with discontinuous coefficients, Stochastic Processes and Their Applications 113 (2004), no. 1, 37-64.

[8] N. V. Krylov and R. Liptser, On diffusion approximation with discontinuous coefficients, Stochastic Processes and Their Applications 102 (2002), no. 2, 235-264.

[9] G. S. Ladde and V. Lakshmikantham, Random Differential Inequalities, Mathematics in Science and Engineering, vol. 150, Academic Press, New York, 1980.

[10] X. Mao, Stochastic Differential Equations and Their Applications, Horwood Publishing Series in Mathematics \& Applications, Horwood, Chichester, 1997.

[11] B. Øksendal, Stochastic Differential Equations. An Introduction with Applications, 4th ed., Universitext, Springer, Berlin, 1995.

[12] P. Protter, Stochastic Integration and Differential Equations, Applications of Mathematics (New York), vol. 21, Springer, Berlin, 1990.

Nikolaos Halidias: Department of Statistics and Actuarial Science, University of the Aegean, Karlovassi 83200, Samos, Greece

E-mail address: nick@aegean.gr

P. E. Kloeden: Fachbereich Mathematik, Johann Wolfgang Goethe Universität, 60054 Frankfurt am Main, Germany

E-mail address: kloeden@math.uni-frankfurt.de 


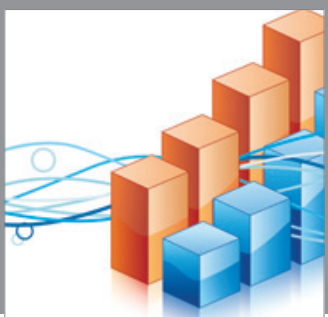

Advances in

Operations Research

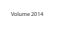

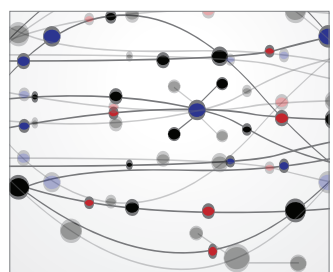

\section{The Scientific} World Journal
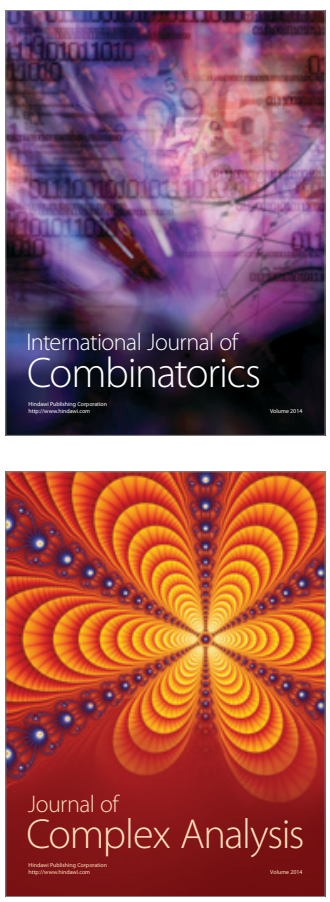

International Journal of

Mathematics and

Mathematical

Sciences
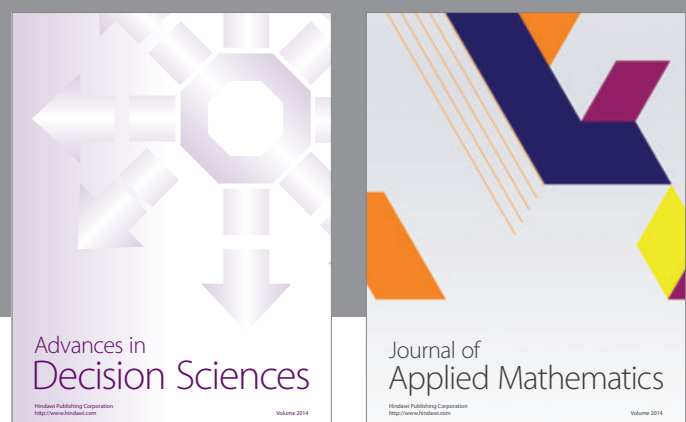

Journal of

Applied Mathematics
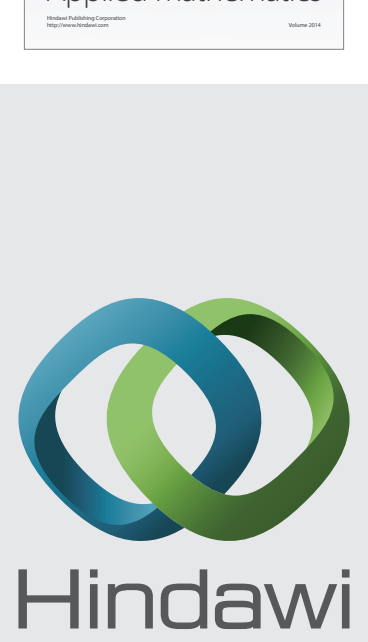

Submit your manuscripts at http://www.hindawi.com
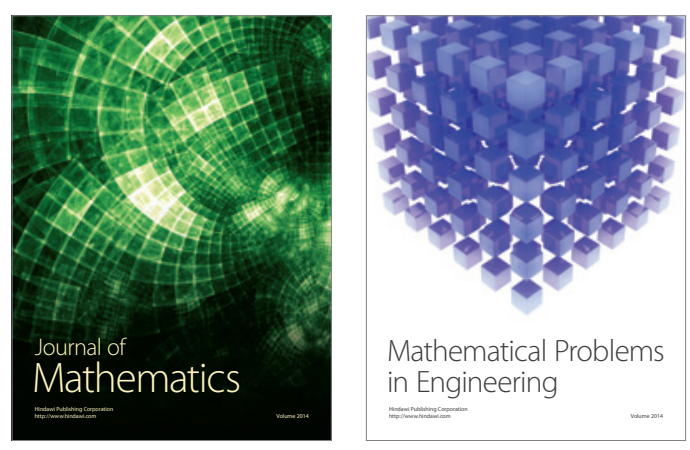

Mathematical Problems in Engineering
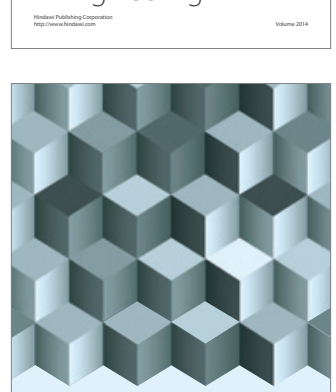

Journal of

Function Spaces
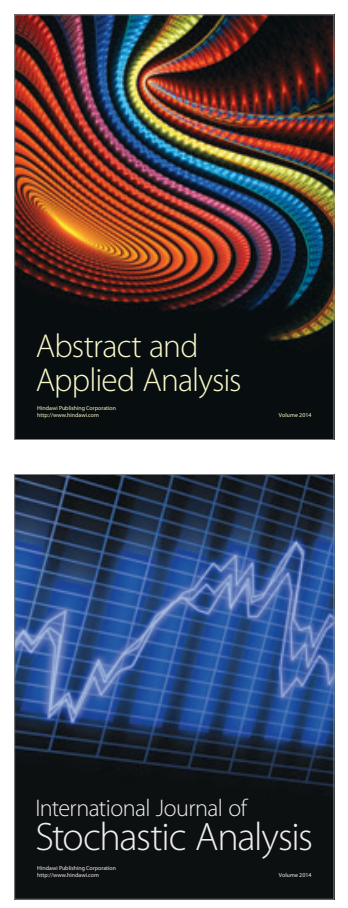

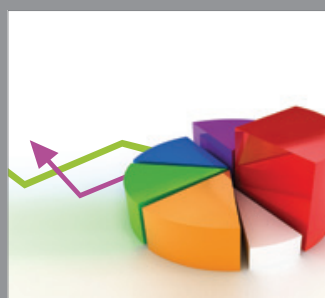

ournal of

Probability and Statistics

Promensencen
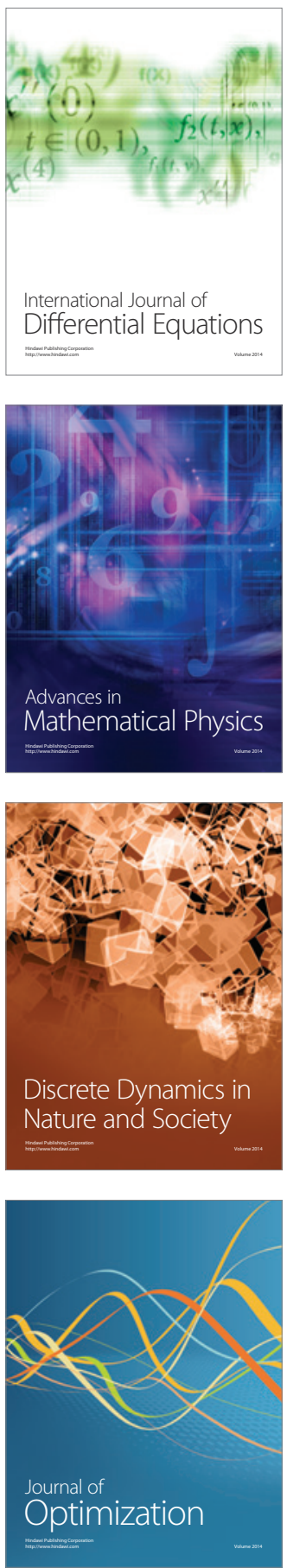